\title{
A Novel Epsilon Near Zero Tunneling Circuit Using Double-Ridge Rectangular Waveguide
}

\author{
Byung-Mun Kim ${ }^{1} \cdot$ Hyeok-Woo Son ${ }^{2} \cdot$ Jae-Pyo Hong ${ }^{3} \cdot$ Young-Ki Cho ${ }^{2, *}$
}

\begin{abstract}
In this paper, an epsilon near zero (ENZ) tunneling circuit using a double-ridge rectangular waveguide (RWG) is proposed for the miniaturization of a waveguide component. The proposed ENZ channel and is located in the middle of the input-output RWG (IORWG). The ratio of the height to the width of the channel waveguide is very small compared to the IORWG. By properly adjusting the ridge dimensions, the tunneling frequency of the proposed ENZ channel can be lowered to near the cut-off frequency of the IORWG. For the proposed ENZ tunneling circuit, the approach adopted for extracting the effective permittivity, effective permeability, normalized effective wave impedance, and propagation constant from the simulated scattering parameters was explained. The extracted parameters verified that the proposed channel is an ENZ channel and electromagnetic energy is tunneling through the channel. Simulation and measurement results of the fabricated ENZ channel structure agreed.
\end{abstract}

Key Words: Double Ridge, Epsilon Near Zero, Rectangular Waveguide, Tunneling.

\section{INTRODUCTION}

Metamaterials, which have been of interest in the fields of physics and engineering for the past several years, are artificial composite materials with negative indexes of permittivity or permeability. Recently, metamaterials with epsilon near zero (ENZ) have been investigated for interesting phenomena, such as supercoupling, transparency, cloaking devices, and highly directive antennas at microwave and optical frequencies [1-3]. The rapid growth and significant attention paid to ENZ materials is because of their ability to achieve a very long wavelength, allowing for the propagation of a "static-like" character of the electromagnetic field.

Silveirinha and Engheta [4] investigated the theory of supercoupling, squeezing wave energy, and field confinement in narrow channels and tight bends using ENZ materials. Previous studies $[5,6]$ have experimentally demonstrated ENZ materials that exhibit supercoupling and energy squeezing using a microwave waveguide. These effects can be applied to a waveguide bend, an antenna matching circuit, a microwave filter, and a dielectric sensing application [1, 7-9]. These technologies have a potential advantage for miniaturization because the electromagnetic energy is tunneled at the specific frequency through a narrow waveguide regardless of the total length of the ENZ channel. Radiation loss can be reduced, as compared with other metamaterials, because these technologies are based on a microstrip and waveguide shape. The tunneling frequency is near the cut-off frequency of the dominant mode in the waveguide. In order to minimize the size of a waveguide device, an ENZ channel can

Manuscript received February 24, 2014 ; Revised March 11, 2014 ; Accepted March 13, 2014. (ID No. 20140224-012J)

${ }^{1}$ Division of IT Cooperative Systems, Gyeongbuk Provincial College, Yecheon, Korea.

${ }^{2}$ School of Electronics Engineering, Kyungpook National University, Daegu, Korea.

${ }^{3}$ Department of Electronics Engineering, Kyungil University, Gyeongsan, Korea.

"Corresponding Author: Young-Ki Cho (e-mail: ykcho@ee.knu.ac.kr)

This is an Open-Access article distributed under the terms of the Creative Commons Attribution Non-Commercial License (http://creativecommons.org/licenses/by-nc/3.0) which permits unrestricted non-commercial use, distribution, and reproduction in any medium, provided the original work is properly cited.

(c) Copyright The Korean Institute of Electromagnetic Engineering and Science. All Rights Reserved. 
be used by a double-ridge rectangular waveguide (RWG) that has a lower cut-off frequency, instead of a general RWG $[10,11]$.

In this paper, we introduce an ENZ tunneling circuit using a double-ridge RWG and we explain the structure of the proposed ENZ channel. The cut-off frequency of the double-ridge RWGs, including a general RWG, is calculated. For the ENZ tunneling circuit using a RWG and a double-ridge RWG, scattering parameters were obtained using the commercial finite element method simulator, Ansoft high-frequency structure simulator (HFSS) software [12]. We discuss in detail two types of transmission resonance phenomena, such as zeroth-order resonance (ZOR) and FabryPerot (FP) resonance, from the results obtained in the simulations. We also explain the approach adopted for extracting the constitutive parameters (effective permittivity, permeability, wave impedance, and propagation constant) of the ENZ channel from the scattering parameters using the retrieval method based on an RWG [13].

Using the extracted constitutive parameters, we explain that the proposed ENZ tunneling circuit using a doubleridge RWG is an ENZ channel, and we discuss, in terms of impedance matching, what electromagnetic energy is tunneled at the tunneling frequency. The measured results of the fabricated ENZ tunneling circuit were quite similar to simulated results, with tunneling frequencies being 9.169 $\mathrm{GHz}$ (simulated) and $9.172 \mathrm{GHz}$ (measured).

\section{Structure of the Proposed ENZ Tunneling Circuit and the Resonance Properties}

\section{Description of the Structure of the Proposed ENZ Channel}

Fig. 1 shows the geometrical structure of the proposed ENZ tunneling circuit, where the ENZ channel is centrally located in the input-output RWG (IORWG, WR-90) with a cross section of $a \times b\left(\mathrm{~mm}^{2}\right)$. The ENZ channel is composed of a double-ridge RWG with a ridge width $w_{r}$ and a ridge gap $h_{g}$ in a rectangular waveguide $w_{c} \times h_{c}\left(\mathrm{~mm}^{2}\right)$. The length of the proposed ENZ channel is $d$.

The ratio of the height of the IORWG to that of the proposed ENZ channel is very high, given as follows:

$$
\frac{b}{h_{c}}>>1
$$

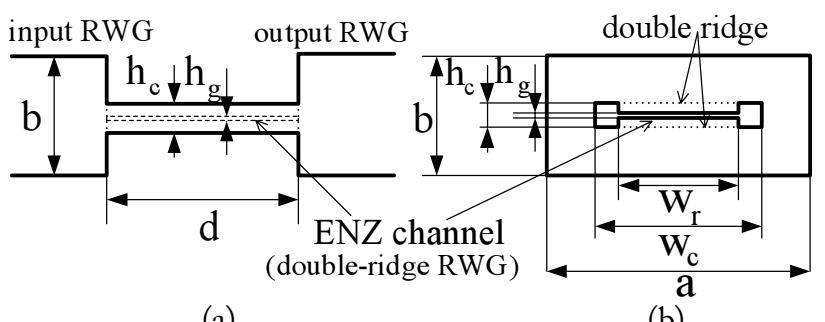

(a)

(b)

Fig. 1. Geometrical parameters of the proposed epsilon near zero (ENZ) tunneling circuit. (a) Lateral side view and (b) cross-sectional view. RWG = rectangular waveguide.
Compared to RWGs, ridge waveguides $[10,11]$ have the advantages of a wide fundamental-mode operation bandwidth, a low cut-off frequency, and low wave impedance. In the ENZ channel, the cut-off frequencies of the RWG and the double-ridge RWG are as follows:

$$
\begin{gathered}
f_{c r}=\frac{c_{0}}{2 w_{c}} \\
f_{c d}=\frac{c_{0}}{2} \frac{1}{w_{c}-w_{r}}[1+A+B]^{-\frac{1}{2}}
\end{gathered}
$$

with

$$
A=\frac{4}{\pi}\left\{\left(1+0.2 \sqrt{\frac{h_{c}}{w_{c}-w_{r}}}\right) \cdot \frac{h_{c}}{w_{c}-w_{r}} \ln \operatorname{cosec}\left(\frac{\pi h_{g}}{2 h_{c}}\right)\right\}
$$

and

$$
B=\left(2.45+0.2 \frac{w_{r}}{w_{c}}\right) \cdot \frac{w_{r} h_{c}}{\left(w_{c}-w_{r}\right) h_{g}}
$$

where $c_{0}$ is the speed of light in free-space, and $\ln$ is the natural logarithm. As mentioned in Eq. (2), the cut-off wavelength of an RWG is equal to twice the waveguide width $w_{c}$. We can estimate the cut-off frequency of the doubleridge RWG in Eq. (3). To calculate accurately the cut-off frequency of the double-ridge waveguide, the transverse resonance method (TRM) is used [14]. As seen in Fig. 2, the cut-off frequency is obtained by using the TRM under the initial value of the approximated cut-off frequency from Eq. (3).

The cut-off frequency of the ENZ channel without the double-ridge is $10.338 \mathrm{GHz}$. As the gap of the ridge $h_{g}$ de-

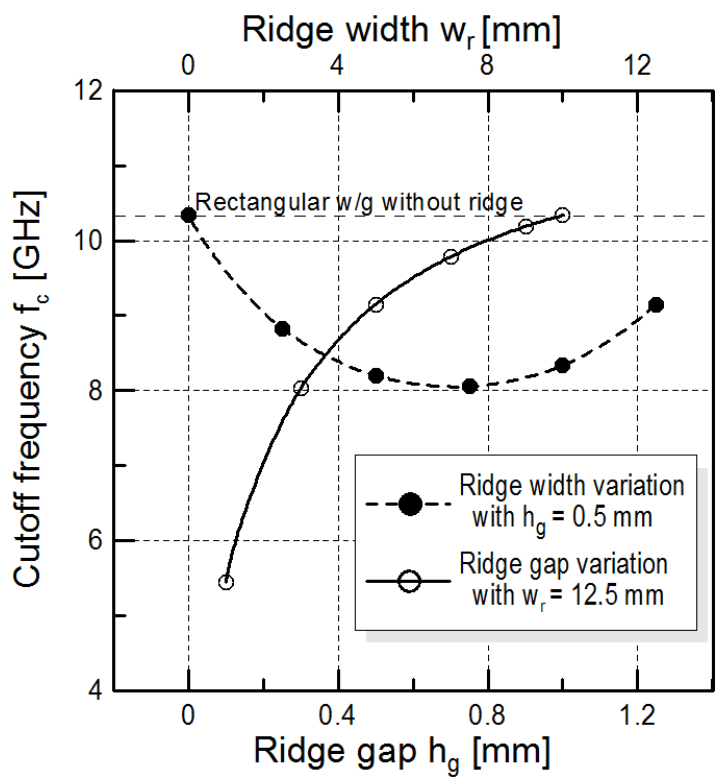

Fig. 2. Cut-off frequency against ridge variation $\left(w_{c}=14.5 \mathrm{~mm}, h_{g}\right.$ $=1 \mathrm{~mm})$ 
creases to $0.1 \mathrm{~mm}$ and the width of the ridge $w_{r}$ is maintained at $12.5 \mathrm{~mm}$, the cut-off frequency of the ENZ channel decreases to $5.446 \mathrm{GHz}$. This indicates that the cut-off frequency becomes low and returns to high when the width of the ridge $w_{r}$ increases to $12.5 \mathrm{~mm}$, keeping the gap of the ridge $h_{g}$ equal to $0.5 \mathrm{~mm}$. Consequently, because the cut-off frequency of the ENZ channel is reduced to less than the cut-off frequency of the IORWG at $6.557 \mathrm{GHz}$, we can appropriately change the structure of the ridge according to the purposes of the ENZ channel.

The guide wavelength $\lambda_{g}$, propagation constant $k_{g}$, phase velocity $v_{p}$, and the characteristic impedance $Z_{r}$ in the ENZ channel are given as follows $[14,15]$ :

$$
\begin{gathered}
\lambda_{g}=\frac{\lambda_{0}}{\sqrt{1-\left(\frac{\lambda_{0}}{\lambda_{c}}\right)^{2}}} \\
k_{g}=2 \pi / \lambda_{g} \\
v_{p}=f \lambda_{g}=2 \pi f / k_{g} \\
Z_{r}=Z_{r}(\infty) \cdot\left(\lambda_{g} / \lambda_{0}\right)
\end{gathered}
$$

where $\lambda_{0}$ is the wavelength in free space, $\lambda_{c}$ is the cut-off wavelength of a waveguide, and $Z_{r}(\infty)$ is the characteristic impedance of the ENZ channel at infinite frequency. At the cut-off frequency $\lambda_{c}=\lambda_{0}$, the guide wavelength is infinite, and the guide wavenumber is zero. This suggests that the electromagnetic fields remain almost unchanged along the longitudinal direction because they are similar to the static electromagnetic fields. Because the characteristic impedance of the ENZ channel is significantly increased around the cut-off frequency, the characteristic impedance of the ENZ channel is equal to the characteristic impedance of the IORWG. This implies that tunneling phenomena are observed to occur when two characteristic impedances are equal. The tunneling frequency is near the cut-off frequency of the ENZ channel.

\section{Scattering Parameters and Extraction of Constitutive Para- meters}

Let us consider the reflection and transmission properties of the ENZ channel using an RWG and a double-ridge RWG. First, to obtain scattering parameters, the ENZ channel using an RWG at an operating frequency of 10.370 $\mathrm{GHz}$ was created to test tunneling phenomena with a fullwave simulator, based on the finite element method, Ansoft HFSS. The results were processed by de-embedding from each port of the IORWG to the interfaces of the ENZ channel. The IORWG uses a standard WR-90 RWG. Table 1 lists the dimensions of the standard RWG and the proposed ENZ channel waveguide applied to a resonator of near-field microwave microscopy. The cut-off frequency $f_{c r}$ and the cut-off wavelength $\lambda_{c r}$ of this ENZ channel are $10.338 \mathrm{GHz}$
Table 1. Dimensions of the proposed epsilon near zero (ENZ) channel waveguide

\begin{tabular}{cc|cc}
\hline Parameter & Value & Parameter & Value \\
\hline$a$ & $22.86 \mathrm{~mm}$ & $w_{r}$ & $12.5 \mathrm{~mm}$ \\
$b$ & $10.16 \mathrm{~mm}$ & $h_{g}$ & $0.5 \mathrm{~mm}$ \\
$w_{c}$ & $14.5 \mathrm{~mm}$ & $d$ & $40 \mathrm{~mm}$ \\
$h_{c}$ & $1.0 \mathrm{~mm}$ & - & - \\
\hline
\end{tabular}

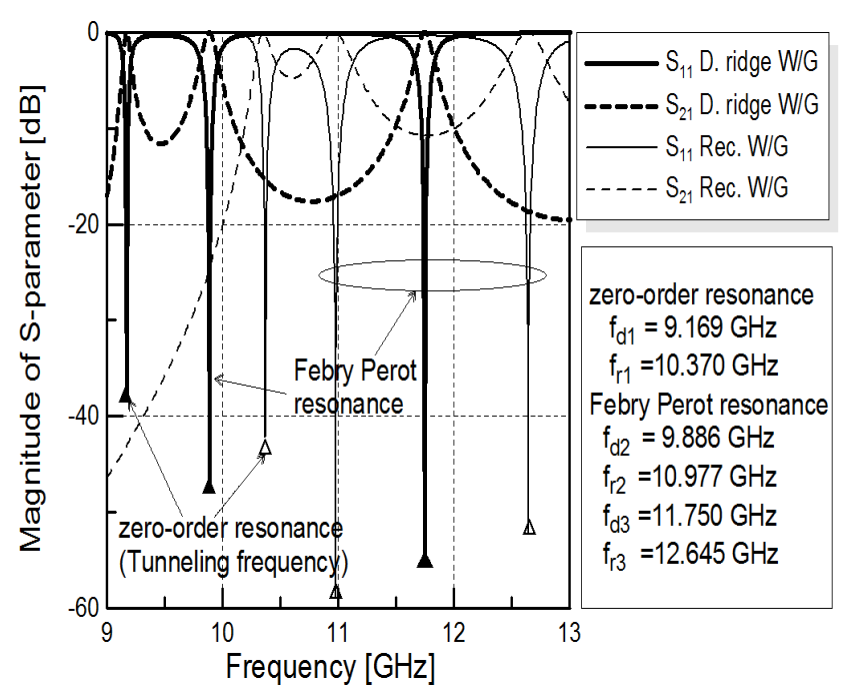

Fig. 3. Simulation results of the proposed epsilon near zero (ENZ) channel. $\mathrm{W} / \mathrm{G}=$ waveguide.

and $29.0 \mathrm{~mm}$, respectively. To reduce the tunneling frequency in the rectangular waveguide, a double-ridge RWG was used, as shown in Fig. 1. Fig. 3 shows the simulation results of the ENZ channel using the RWG and the double-ridge RWG for a frequency range of 9.0 to $13.0 \mathrm{GHz}$. These results imply that the two types of transmission resonances, ZOR and FP, have been observed in the proposed ENZ channel. The tunneling frequency of the ENZ channel using the RWG and the double-ridge RWG are 10.370 and $9.169 \mathrm{GHz}$, respectively.

Because the frequencies are near the cut-off frequency of each ENZ channel (10.336 and $9.152 \mathrm{GHz}$ ), the guide propagation constant is close to zero, implying that the length of the ENZ channel is insensitive because the guide wavelength is very long. These resonances are of the ZOR type. The ZOR mainly depends on the transverse cross-sectional shape, which is related to the cut-off frequency of an ENZ channel. Other resonant frequencies of the ENZ channel using the RWG and the double-ridge RWG are $\left(f_{r 2}=\right.$ $\left.10.977 \mathrm{GHz}, f_{r 3}=12.645 \mathrm{GHz}\right)$ and $\left(f_{d 2}=9.886 \mathrm{GHz}, f_{d 3}\right.$ $=11.750 \mathrm{GHz}$ ). These resonances are of the FP resonance type. The FP resonances occur when the length of the ENZ channel is around an integer number of the half-guide wave length, $n \frac{\lambda_{g}}{2}(n=1,2,3, \ldots)$. The wavelengths of the channel using the RWG and the double-ridge RWG are 81.2 
$\mathrm{mm}\left(\right.$ at $f_{r 2}=10.977 \mathrm{GHz}$ ) and $80.2 \mathrm{~mm}$ (at $f_{d 2}=9.886$ $\mathrm{GHz}$ ), respectively. The length of the proposed ENZ channel $d$ is $40 \mathrm{~mm}$. The length of the ENZ channel at the resonance frequencies does not exactly agree with $n \frac{\lambda_{g}}{2}$, because of the shunt susceptance for the stored electromagnetic energy at the interface of the IORWG and the ENZ channel.

The effective permittivity $\varepsilon_{e f f}$; permeability $\mu_{\text {eff }}$, wave impedance $Z_{e f f}$, and propagation constant $\gamma_{g d}$ can be extracted using the retrieval method from the simulated scattering $\mathrm{pa}^{-}$ rameters $[13,16]$. The scattering parameters can be expressed as functions of the reflection coefficient $\Gamma$ and the transmission coefficient $T$ as follows:

$$
\begin{aligned}
& S_{11}=\frac{\Gamma\left(1-T^{2}\right)}{1-\Gamma^{2} T^{2}}, \\
& S_{21}=\frac{T\left(1-\Gamma^{2}\right)}{1-\Gamma^{2} T^{2}} .
\end{aligned}
$$

Eqs. (10) and (11) can be solved for

$$
\Gamma=K \pm \sqrt{K^{2}-1}
$$

with

$$
K=\frac{S_{11}^{2}-S_{21}^{2}+1}{2 S_{11}}
$$

and

$$
T=\frac{S_{11}+S_{21}-\Gamma}{1-\left(S_{11}+S_{21}\right) \Gamma} .
$$

In Eq. (12), the sign of the square root has to be chosen such that $|\Gamma| \leq 1$. The propagation constants $\gamma_{g r}$ and $\gamma_{g d}$ of the IORWG and the ENZ channel using the double-ridge RWG can be calculated as follows:

$$
\begin{gathered}
\gamma_{g r}=j 2 \pi \sqrt{\frac{1}{\lambda_{0}^{2}}-\frac{1}{\lambda_{c r}^{2}}} \\
\gamma_{g d}=[\ln (1 / T)] / d
\end{gathered}
$$

where $\lambda_{c r}$ is the cut-off wavelength of the IORWG. The effective permittivity $\varepsilon_{\text {eff }}$ and permeability $\mu_{\text {eff }}$ can be determined using Eqs. (12), (15), and (16) as follows:

$$
\begin{gathered}
\varepsilon_{e f f}=\frac{\gamma_{g d}(1-\Gamma)}{\lambda_{g r}(1+\Gamma)}, \\
\mu_{e f f}=\frac{\gamma_{g d}(1-\Gamma)}{\lambda_{g r}(1+\Gamma)} .
\end{gathered}
$$

The effective wave impedance of the ENZ channel is

$$
Z_{e f f}=Z_{o r} \sqrt{\frac{\mu_{e f f}}{\varepsilon_{e f f}}}=Z_{o r} \frac{(1+\Gamma)}{(1-\Gamma)},
$$

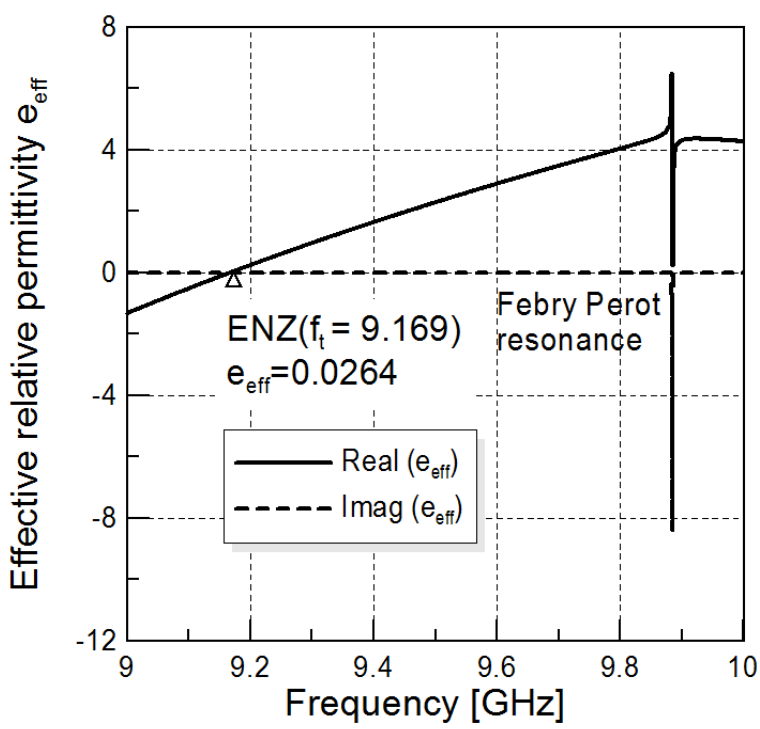

(a)

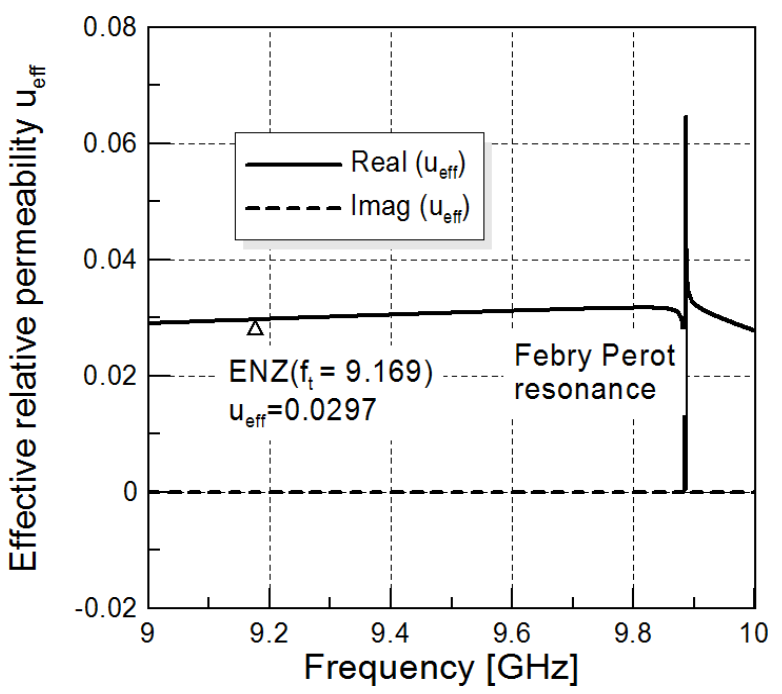

(b)

Fig. 4. Effective permittivity and permeability for the proposed epsilon near zero (ENZ) channel. (a) Effective permittivity $\varepsilon_{\text {eff }}$ and (b) effective permeability $\mu_{\text {eff }}$.

where $Z_{0 r}$ is the wave impedance of the IORWG. Then from Eqs. (19) and (16), the reflection coefficient and transmission coefficient are finally determined as

$$
\Gamma=\frac{Z_{\text {eff }}-Z_{\text {or }}}{Z_{\text {eff }}+Z_{\text {or }}}
$$

and

$$
T=e^{-\gamma_{g d} d} .
$$

At the tunneling frequency $Z_{e f f}=Z_{0 r}$, the reflection coefficient and transmission coefficient have to be "0" and "1", respectively.

The extracted effective permittivity $\varepsilon_{\text {eff; }}$, permeability $\mu_{\text {eff }}$; normalized wave impedance $z_{\text {eff }}$, and propagation constant $\gamma_{g d}$ are shown in Figs. 4 and 5. 


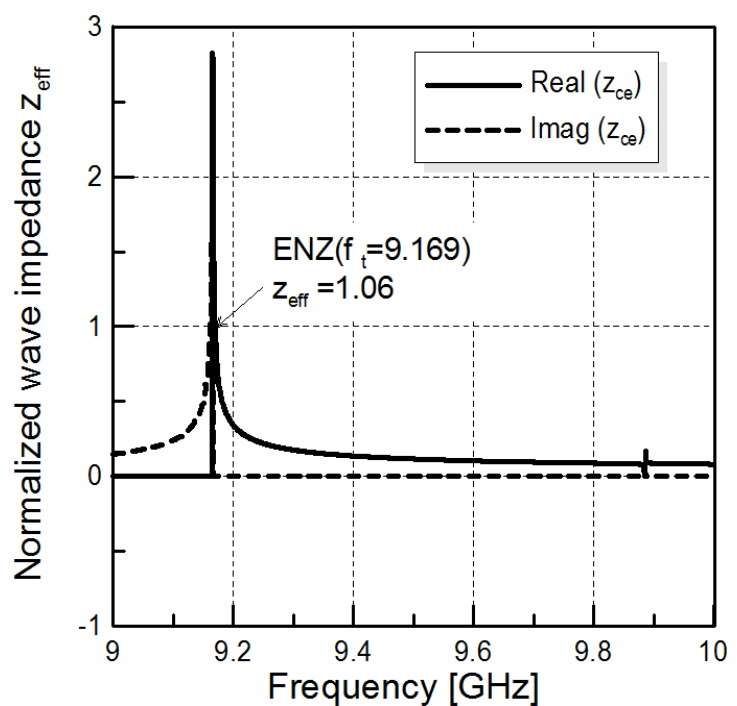

(a)

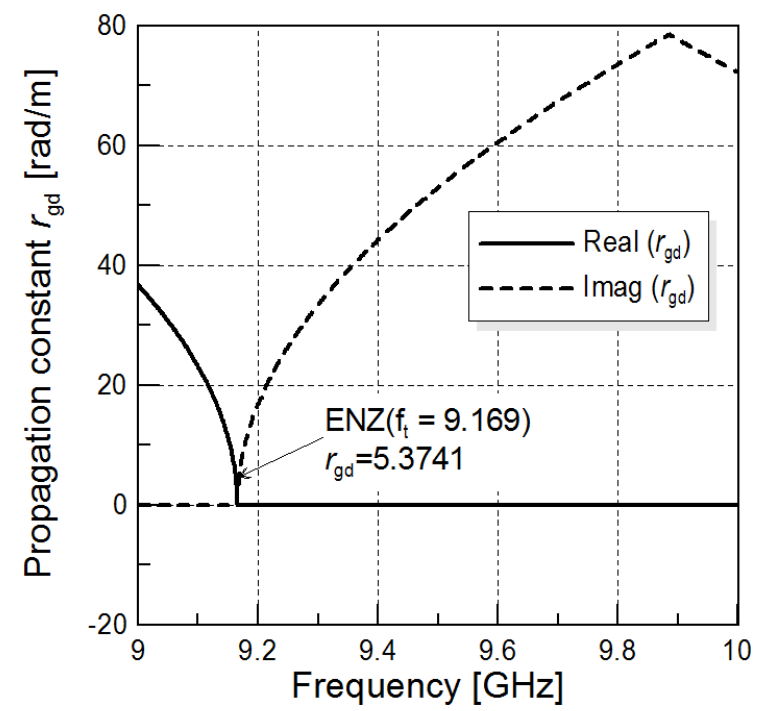

(b)

Fig. 5. Normalized wave impedance $z_{\text {eff }}$ and propagation constant $\gamma_{g d} . \mathrm{ENZ}=$ epsilon near zero.

In Fig. 4(a), we note that the proposed structure is the ENZ channel, because the real value of the effective permittivity $\varepsilon_{\text {eff }}$ is 0.0264 at the tunneling frequency $(9.169 \mathrm{GHz})$. The real value of the effective permeability is 0.0297 in Fig. 4(b). The normalized wave impedance $z_{\text {eff; }}$, which is normalized with the wave impedance $Z_{0 r}$ of the IORWG, is 1.06 in Fig. 5(a). The effective wave impedance of the proposed ENZ channel using the double-ridge RWG is typically lower than that of the IORWG, but that of the former was significantly increased around the cut-off frequency. Then, the effective wave impedance was equal to the wave impedance of the IORWG at the tunneling frequency. Thus, good impedance matching was observed, as with [17], implying that the tunneling phenomena of electromagnetic energy occurred in the ENZ channel. The propagation constant $\gamma_{g d}$ of the ENZ channel as shown in Fig. 4(b) is zero at the cut-off

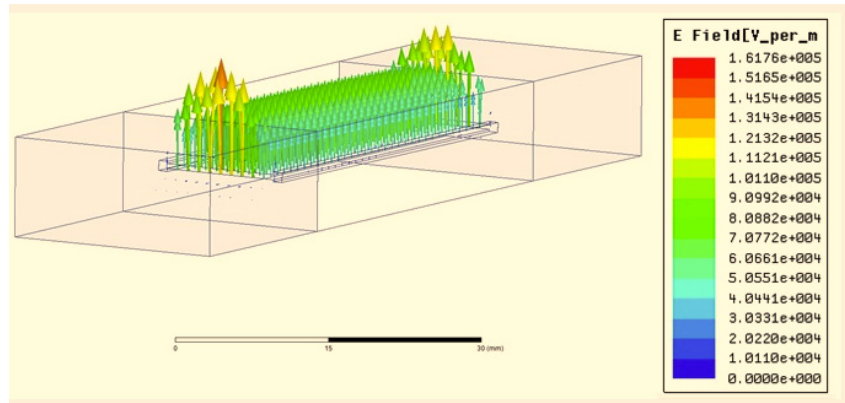

Fig. 6. Electric field distribution along the proposed epsilon near zero (ENZ) channel.

frequency of $5.3741(\mathrm{rad} / \mathrm{m})$ and the guide wavelength is $1.1691 \mathrm{~m}$ at the tunneling frequency $(9.169 \mathrm{GHz})$. The phase variation in the electric fields in the ENZ channel using a double-ridge waveguide is approximately $0.2150 \mathrm{rad}$ $\left(12.3186^{\circ}\right)$. It is clear that the effective permittivity has a value of zero near the tunneling frequency, and the propagation constant is near zero, which satisfies the conditions for ENZ behavior.

Fig. 6 shows the field distribution along the channel at the tunneling frequency, which exhibits little phase shift along the channel.

\section{Fabrication And Measurements of the ENZ CHANNEL}

Aluminum was used for the ENZ channel circuit. The ENZ channel using the double-ridge RWG was manufactured using a wire electro-discharge machine, as shown in Fig. 7. The IORWGs, which were the size of a WR-90 standard waveguide, were connected to the ENZ channel using separated screws. The ENZ tunneling circuit for characteristic measurements was connected to a network analyzer (Anritsu 37397C) through a coaxial waveguide adapter, which differs from the waveguide port used in the simulation to compute the input reflection coefficient $\left(S_{11}\right)$.

This causes small differences between the measured and simulated input reflection coefficients, as shown in Fig. 8. The measured tunneling frequency $f_{m}$ is $9.172 \mathrm{GHz}$ and has a tolerance of $3 \mathrm{MHz}$ in comparison with the theoretical value $\left(f_{d 1}=9.169 \mathrm{GHz}\right)$.

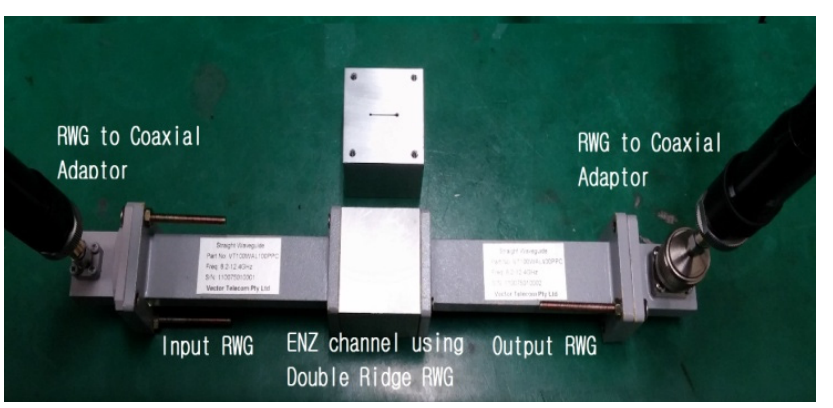

Fig. 7. Photo of the fabricated epsilon near zero (ENZ) channel using the double-ridge rectangular waveguide (RWG). 


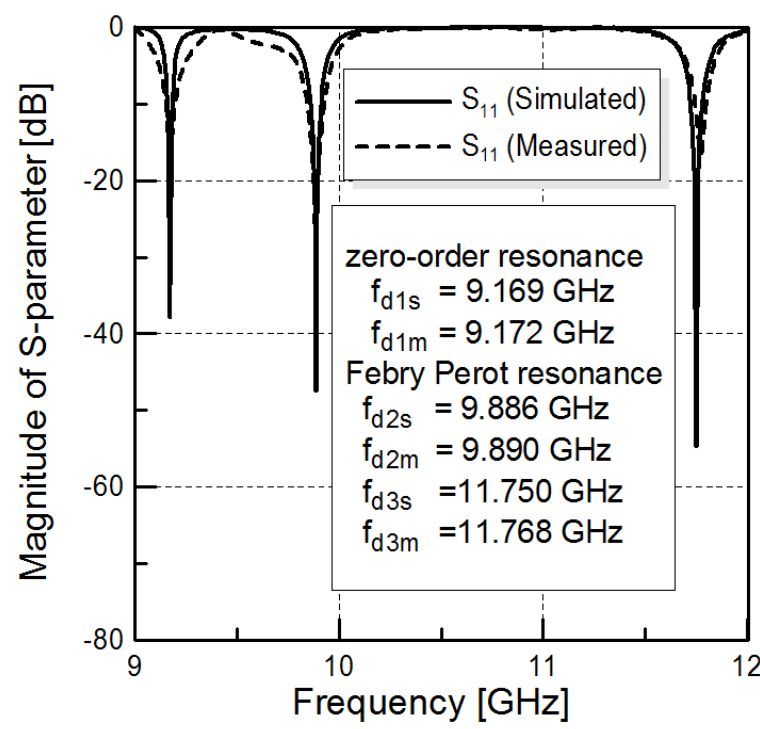

Fig. 8. Simulated and measured scattering parameters $\left(S_{11}\right)$ of the proposed epsilon near zero (ENZ) tunneling circuit using a double-ridge rectangular waveguide.

\section{CONCLusions}

In this paper, we proposed an ENZ tunneling circuit using a double-ridge waveguide for the miniaturization and field confinement of waveguide circuits and the characteristics of that system were analyzed. The proposed ENZ channel which was centrally located in a standard RWG, used a narrowed double-ridge RWG. The tunneling frequency of the proposed ENZ channel can be reduced to near the cut-off frequency of the IORWG. From the extracted parameters, the proposed double-ridge waveguide channel must become the ENZ channel because the effective permittivity is zero at the cut-off frequency and close to zero at the tunneling frequency. The normalized effective wave impedance is almost "1" at the tunneling frequency, implying that we have verified that the electromagnetic energy was tunneled. The measured scattering parameters of the proposed ENZ channel agreed with the simulation results, which had tunneling frequencies of $9.169 \mathrm{GHz}$ (simulated) and $9.172 \mathrm{GHz}$ (measured). In the future, we will apply an ENZ channel using a double-ridge waveguide to the probe antenna of a nearfield microwave microscope.

This study was supported by BK21 Plus funded by the Ministry of Education, Korea (21A20131600011) and the Basic Science Research Program through the National Research Foundation of Korea (NRF) funded by the Ministry of Education, Science and Technology (2010-0024647).

\section{REFERENCES}

[1] M. Silveirinha and N. Engheta, "Tunneling of electromagnetic energy through subwavelength channels and bends using $\varepsilon$-near-zero materials," Physical Review Letters, vol.
97, no. 15 , pp. 157403 , Oct. 2006.

[2] A. Alu and N. Engheta, "Achieving transparency with plasmonic and metamaterial coatings," Physical Review E, vol. 72, no. 1, pp. 16623, Jul. 2005.

[3] A. Alu, M. Silveirinha, A. Salandrino, and N. Engheta, "Epsilon-near-zero metamaterials and electromagnetic sources: tailoring the radiation phase pattern," Physical Review B, vol. 75, no. 15, pp. 155410, Apr. 2007.

[4] M. Silveirinha and N. Engheta, "Theory of supercoupling, squeezing wave energy, and field confinement in narrow channels and tight bends using $\varepsilon$ near zero metamaterials," Physical Review B, vol. 76, no. 24, pp. 245109, Dec. 2007.

[5] Y. K. Cho, J. Y. Choi, H. W. Son, and J. H. Ko, "Experimental verification of two kinds of transmission resonance phenomena for a narrow slot in thick conducting screen," Journal of the Korean Physical Society, vol. 63, no. 8, pp. 1554-1558, Oct. 2013.

[6] B. Edwards, A. Alu, M. E. Young, M. Silveirinha, and N. Engheta, "Experimental verification of epsilon-nearzero metamaterial coupling and energy squeezing using a microwave waveguide," Physical Review Letters, vol. 100, no. 3, pp. 033903, Jan. 2008.

[7] A. Alu and N. Engheta, "Antenna matching in $\varepsilon$-nearzero metamaterial channels," in Proceedings of the IEEE International Workshop on Antenna Technology, Santa Monica, CA, 2009, pp. 1-4.

[8] A. Corona-Chavez and T. Itoh, "Bandwidth enhancement of substrate integrated waveguide tunnels by longitudinal resonances," in Proceedings of the Microwave Symposium Digest, Anaheim, CA, 2010, pp 872-875.

[9] A. Alu and N. Engheta, "Dielectric sensing in $\varepsilon$-nearzero narrow waveguide channels," Physical Review B, vol. 78, no. 4, pp. 045102, Jul. 2008.

[10] S. B. Cohn, "Properties of ridge waveguide," Proceedings of the IRE, vol. 35, no. 8, pp. 783-788, Aug. 1947.

[11] J. R. Pyle, "The cutoff wavelength of the TE10 mode in ridged rectangular waveguide of any aspect ratio," IEEE Transactions on Microwave Theory and Techniques, vol. 14, no. 4, pp. 175-183, Apr. 1966.

[12] Ansoft HFSS Software (ver. 10) [Online]. http://ansys-hfss.software.informer.com/.

[13] T. Zwick, A. Chandrasekhar, C. W. Baks, U. R. Pfeiffer, S. Brebels, and B. P. Gaucher, "Determination of the complex permittivity of packaging materials at millimeter-wave frequencies," IEEE Transactions on Microwave Theory and Techniques, vol. 54, no. 3, pp. 10011010, Mar. 2006.

[14] J. Helszajn, Ridge Waveguides and Passive Microwave Components. London: Institution of Electrical Engineering, 2000.

[15] R. E. Collin, Field Theory of Guided Waves. New York, NY: McGraw-Hill, 1960.

[16] G. Lubkowski, R. Schuhmann, and T. Weiland, "Extraction of effective metamaterial parameters by para- 
meter fitting of dispersive models," Microwave and $O p-$ tical Technology Letters, vol. 49, no. 2, pp. 285-288, Feb. 2007.

[17] S. T. Kahng, "Study on the electromagnetic wave prop- agation in the parallel-plate waveguide with the metamaterial ENZ tunnel embedded," Journal of Korean Institute of Electromagnetic Engineering and Science, vol. 20, no. 2, pp. $135-140,2009$.

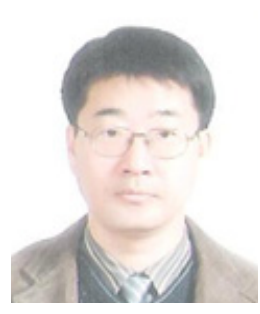

and radar system.
Byung-Mun Kim was born in Waegwan, Korea. He received the B.S. and M.S. degrees in electronic engineering from Kyungpook National University, Daegu, Korea, in 1986 and 1988, respectively. Since 1997, he is a Professor of Division of IT Cooperative Systems of Gyeongbuk Provincial College, Yecheon, and his research areas are antenna and microwave circuits,

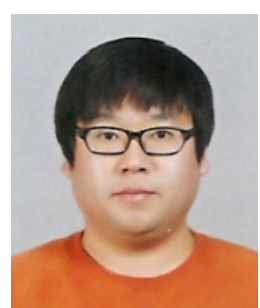

Hyeok-Woo Son was born in Korea in 1983. He received his B.S. and M.S. degrees from the Department of Electronics Engineering of $\mathrm{Ky}^{-}$ ungpook National University, Daegu, Korea, in 2009 and 2011, respectively. He is currently taking his $\mathrm{Ph} . \mathrm{D}$ at Kyungpook National University. His current research interests are electromagnetic theory, metamaterial, antenna design and MRI RF Coil design.

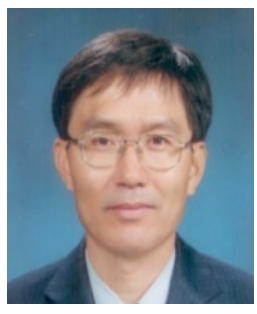

Jae-Pyo Hong was born in Daegu, Korea. He received the B.S. degree in electronic engineering from Kyungpook National University, Daegu, Korea, in 1981, and the M.S. and Ph.D. degrees in electronic Engineering from Kyungpook National University, in 1983 and 1989, respectively. Since 1990, he is a Professor of electronic engineering of Kyungil University, Gyeongsan, and his research areas are antenna and microwave circuits design.

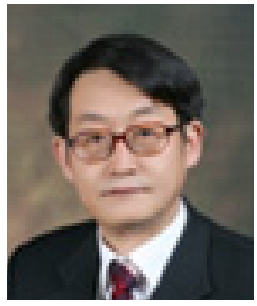

Young-Ki Cho received a B.S. degree in Electrical Engineering from Seoul National University, Seoul, Korea, in 1978. He received M.S. and $\mathrm{Ph} . \mathrm{D}$. degrees in Electrical Engineering from the Korea Advanced Institute of Science and Technology (KAIST), Daejeon, Korea. In 1981, he joined the School of Electrical Engineering and Computer Science at Kyungpook National University, Daegu, Korea. Since 1992, he has been Korea's representative on URSI Commission B, Fields and Waves. In 2008, he became President of the Korean Institute of Electromagnetic Engineering and Science. His research interests include electromagnetic theory, scattering problem, antenna, and RF devices. 